\title{
Infection of Calomys callosus (Rodentia Cricetidae) with Strains of Different Trypanosoma cruzi Biodemes: Pathogenicity, Histotropism, and Fibrosis Induction
}

\author{
Isis Fernandes Magalhães-Santos, Márcia Maria Souza, Carolina Silva Costa Lima, \\ Sonia G Andrade ${ }^{+}$
}

Laboratório de Doença de Chagas Experimental, Autoimunidade e Imunologia Celular e Laboratório de Patologia Experimental, Centro de Pesquisas Gonçalo Moniz-Fiocruz, Rua Valdemar Falcão 121, 40295-001 Salvador, BA, Brasil

The influence of different Trypanosoma cruzi biodemes on the evolution of the infection and on the histopathological lesions of the heart and skeletal muscles, during the experimental infection of Calomys callosus, was investigated. Three groups of $\mathrm{C}$. callosus were infected, respectively, with parasite strains representative of three different Biodemes: Type I (Y strain), Type II (21 SF strain), and Type III (Colombian strain). For each group, normal C. callosus were also used as controls. Marked differences have been detected in the responses of $\mathrm{C}$. callosus to the infection with the three strains in this model. The strains Types I and II ( $Y$ and 21 SF) determined moderate lesions, mostly in the myocardium, with low parasitism, a rapid course, and total regression of the lesions by the 60th day of infection. Differently, Type III strain (Colombian), was more pathogenic for C. callosus and induced necrotic-inflammatory lesions in skeletal muscles and myocardium, in correspondence to intracellular parasitism. Proliferation of fibroblasts and amorphous matrix deposits, followed by interstitial fibrosis were present. Progressive regression of the inflammatory changes and collagen deposits occurred spontaneously. The progression and regression of both inflammation and fibrosis induced by the Colombian strain were further submitted to quantitative evaluation by morphometry. Results of the morphometric studies presented good correlation with the histopathological findings. The results confirm the importance of the different biodemes in the determination of tissue lesions and the peculiarities of response of $\mathrm{C}$. callosus to infection with $\mathrm{T}$. cruzi.

Key words: Calomys callosus - Trypanosoma cruzi - biodemes - histopathology - morphometry fibrosis - inflammation

Since the initial studies of Carlos Chagas (1909) different animal species as dogs, guinea-pigs, and monkeys (Callithrix penicillata) has been used with the objective of to investigate the evolution of infection and the susceptibility of different vertebrate hosts to Trypanosoma cruzi. Early studies by Brumpt and Pirajá da Silva (1912) in the guinea-pig, have indicated the differences of behavior of samples of $T$. cruzi from different origins, one from Minas Gerais, isolated by Chagas and one from Mata do São João, Bahia, isolated by Pirajá da Silva.

The patterns of the lesions determined in different experimental models by the inoculation with different strains of $T$. cruzi have varied on the dependence of the combined influence of parasite strain and animal species. In the murine model, a diversity of responses were also observed in relation to high or low susceptibility of the inbred strains used (Andrade et al. 1985). Comparative studies on the influence of several strains of $T$. cruzi in Calomys callosus and mouse infections have been previously performed by Borges et al. (1992). They used inoculation with the Y strain from human origin, and with

Financial support: Fundação de Amparo à Pesquisa do Estado da Bahia, process 19.571.049.3383

${ }^{+}$Corresponding author. Fax: +55-71-356-4292. E-mail: sgandrade @ cpqgm.fiocruz.br

Received 17 February 2004

Accepted 26 May 2004 the Costalimai and M226 strains, from silvatic origin. Contrary to the murine model, C. callosus controlled the parasitemia and showed regression of the lesions within a few days. However, when the infection was caused by the F or Colombian strains (Type III Biodeme strains), a high susceptibility was disclosed, with intense parasitism of myocardium and skeletal muscles and intense inflammatory and fibrotic lesions, which spontaneously subsided (Andrade et al. 1994, Magalhães-Santos et al. 2002).

Considering the peculiarities of $C$. callosus responses to T. cruzi from different biodemes, belonging to Types I, II, and III (Andrade 1974, Andrade \& Magalhães 1997), the patterns of infection by T. cruzi, and the histopathological lesions induced in this host, have herein been investigated.

In a previous study, the process of fibrogenesis and collagen resorption, determined by the Colombian strain of $T$. cruzi in the $C$. callosus model, has been investigated with the objective of characterizing the components of the extracellular matrix involved as well as to determine the genetic types of collagens predominant in this process (Magalhães-Santos et al. 2002). Results of that investigation indicated the presence of the collagens types I, III, and IV. Regardless of the genetic type of collagen, the modulation of the inflammatory process and the control of parasite multiplication resulted in the resorption of collagen.

For a better evaluation of the inter-relationship between these processes, a morphometric evaluation of the collagen deposits and inflammation areas of the myocar- 
dium and skeletal muscles of Colombian strain-infected C. callosus was performed.

In the present study, it was observed that the strains of type I (Y) and Type II (21SF) do not determine a significant increase in collagen deposits as compared with normal controls. The infection is soon controlled, leading to the regression of inflammation and parasitism. In these cases morphometric evaluation has not been performed, considering the patent regression of inflammatory infiltrates and the scarcity of interstitial deposits.

Results indicated that the strains of three different biodemes behave differently in the $C$. callosus infection, which confirms the importance of parasite strain characteristics on the determination of lesions in the vertebrate host. A peculiar response of $C$. callosus to infection with Biodeme Type III, related to fibrogenic process, was also detected and quantitatively evaluated.

\section{MATERIALS AND METHODS}

Experimental animals - Ninety-five C. callosus raised in the animal facilities at Centro de Pesquisas Gonçalo Moniz-Fiocruz were used in this study. The experimental animals were approximately 60-day old males, weighing 20-30 g. Their maintenance and handling complied with the guidelines for the human use of laboratory animals.

Strains of T. cruzi - Twenty five C. callosus were infected with the $Y$ strain of $T$. cruzi (Biodeme Type I, Zimodeme Z2b); 25 were infected with the $21 S F$ strain (Biodeme Type II, Z2, T. cruzi II ); 30 calomys infected with the Colombian strain (Biodeme Type III, Z1, T. cruzi I). For each infected group, 5 normal controls were studied.

Inoculum - Consisted of $10^{5}$ trypomastigote forms per animal. Parasites were obtained from the blood of infected mice, washed three times with PBS by centrifugation, and inoculated intraperitoneally.

Mortality - Cumulative mortality was evaluated during the course of infection for all the experimental groups.

Parasitemia - Five animals were evaluated three days a week, by counting the parasite numbers in 50 high power (x 400) fields by microscopic examination of peripheral blood under coverslips ( $22 \times 22 \mathrm{~mm})$.

Histopathology - For histopathological study, three C. callosus per point were sacrificed by exsanguination under anesthesia, 7, 10,15, 25,30,40, days post-infection for the $Y$ strains (Type I) and 10, 15, 25, 30, 40, and 60 days for the 21 SF strain (Type II).

As for the Colombian strain (Type III), sacrifice occurred at $15,20,25,30,35,40,45,60,65$, and 70 days postinfection.

Fragments of the heart and skeletal muscles were fixed in formalin, paraffin-embedded and the $5 \mu \mathrm{m}$-thick sections obtained were stained with hematoxylin and eosin (H\&E).

Picro-Sirius staining method was used for collagen identification in paraffin sections of the heart and skeletal muscle with Rot-Sirius 3 (Chroma Gessellschaft) in a saturated aqueous solution of picric acid and fast green as a counterstain (Junqueira et al. 1979). Sections were examined with direct light and with bright field light optics using a Zeiss photo microscope.
Morphometry - Quantitative evaluation of inflammation and fibrosis, stained with $\mathrm{HE}$ and Picrossirius respectively, were performed in heart and skeletal muscle of $C$. callosus, infected with the Colombian strain, at different evolutive phases of the infection: early phase, 25 days; intermediary phase, 30 days; advanced phase, 40 days; and late phase, 60 days. For the evaluation of both inflammation and fibrosis, a total area of $60 \mathrm{~mm}^{2}$ was analyzed per animal (five field of $12 \mathrm{~mm}^{2}$ ), being three animals from each group. For all semi-automated morphometry, an optical microscope (Zeiss) was used, and the images were captured and evaluated using the program Axio Vision 3.1 (1998-2002), Carl Zeiss Vision GmbH (Zeppelinstrasse 485399 Munchen-Hallbergnoss, Germany).

Evaluation of inflammatory infiltrates - The degree of inflammation in sections of myocardium and skeletal muscles was evaluated by examining inflammatory infiltrates in H\&E stained sections from five microscopic fields, not successively, using an 10X ocular and a $40 \mathrm{X}$ objective. The sectional areas of inflammation were directly measured and the total number of cells of the examined area calculated.

Evaluation of fibrosis - The degree of fibrosis in sections of the myocardium and skeletal muscle were evaluated by examining five selected areas of fibrosis, SiriusRed stained, corresponding to microscopic fields 400X, excluding the normal fibrous structures of the heart and the interstitial septa of the skeletal muscles. The sectional areas of the fibrous tissue were directly measured and calculated as a percentage of the total area examined.

Statistical analysis - ANOVA non-parametric and Dunnett's multiple comparative tests were applied. Results were considered significant when determinations reached $\mathrm{p}<0.05$.

\section{RESULTS}

Parasitemia - Evolution of parasitemia is shown in Fig. 1 for the several groups. Comparing the three strains, the Y strain determined the highest and earliest peak of parasitemia by the 10th day of infection; the 21SF strain determined the lowest peak at the 12th day; the Colombian strain showed intermediate parasitemic peak by the 14th day of infection.

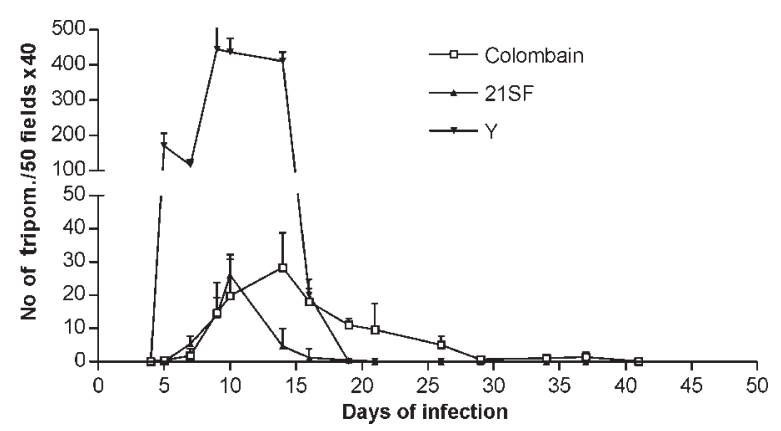

Fig. 1: parasitemic profiles detected in the peripheral blood of Calomys callosus infected with the strains Y (Biodeme Type I); 21SF (Biodeme Type II), and Colombian (Biodeme Type III). Comparing the three strains, the $\mathrm{Y}$ strain determined the highest and earliest peak (10th day of infection); the 21SF strain determined the lowest peak, occurring at the 12th day; the Colombian strain showed an intermediate parasitemic peak by the 14th day of infection. 
Histopathological lesions

Y strain (Biodeme Type I): heart - C. callosus infected with the Y strain showed an evolutive parasitism of the myocardium, from the 7th day of infection, increasing rapidly until the 10th day, with an intense parasitism of myocells. An intense myocarditis was present, with diffuse and focal mononuclear infiltrates, and focal necrosis of cardiac cells. From 15 to 20 days (Fig. 2A) of infection, regression of inflammation and parasitism were seen, with focal mononuclear infiltrates and mild deposits in the connective tissue. Lesions in the myocardium subsided by the 40th day post infection (Fig. 2B).

Skeletal muscles - The sections exhibited normal histology. No inflammation, fibrosis or parasitism was present, and the Picro Sirius staining did not reveal abnormal collagen deposits in the skeletal muscles.

21 SF strain (Biodeme Type II): heart - Lesions in the myocardium were absent until 10 days of infection. From 15 to 30 days of infection, scarce parasites were present with necrosis of cardiac cells as well as mild to moderate focal and diffuse inflammatory infiltration with macrophages and fibroblasts (Fig. 2C). From 35 to 60 days, mild focal inflammatory infiltrates were seen in the myocar- dium, in the absence of parasites (Fig. 2D); the interstitial matrix was scarce, with thin strands of collagen seen by Picro Sirius staining, in 6/15 cases.

Skeletal muscles - Small perivascular mononuclear infiltrates were seen by the 10th day of infection. No parasites were identified in the examined sections. From 15 to 20 days mild focal infiltrates, scarce parasites and focal necrosis of muscle cells were seen. Up to 35 days after infection, perivascular infiltrates of mononuclear cells were present, as well as focal necrosis of muscle cells, without parasites. Fibrotic alterations were absent. Total regression, of the histopathological alterations was registered from 40 to 60 days post infection.

Colombian strain (Biodeme Type III): heart - In the myocardium, mild to moderate diffuse mononuclear infiltration as well as focal infiltration around necrotic cardiac cells were seen from 15 to 20 days, with presence of macrophages and fibroblasts (Figs 3A, B). From 25 to 30 days of infection moderate to intense, diffuse and focal inflammatory infiltrate, with predominance of macrophages and lymphocytes, was present as well as proliferation of fibroblasts and interstitial matrix deposits ( Figs 3C, D). Picro-Sirius staining showed collagen deposits as slen-
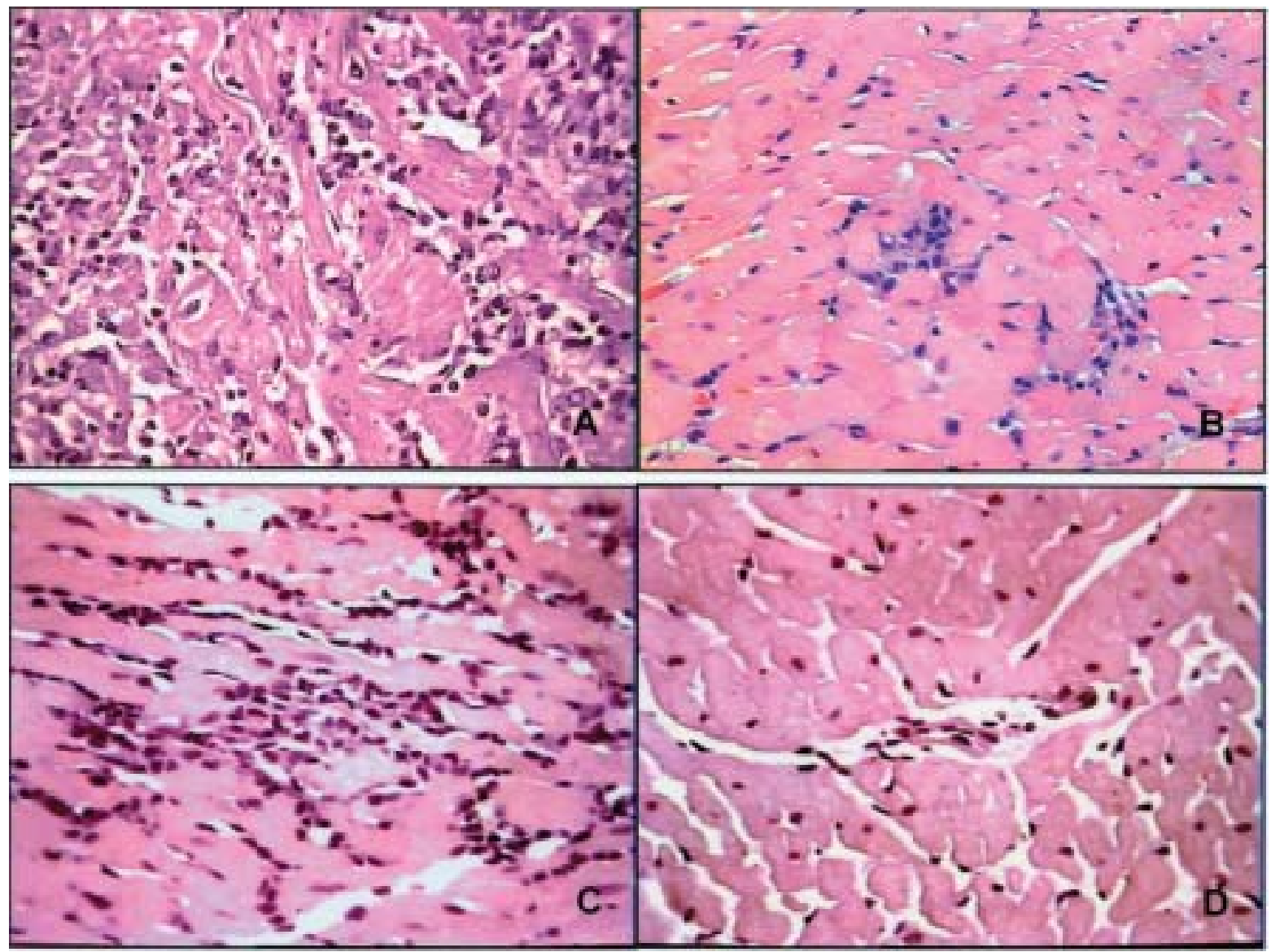

Fig. 2: histopathological lesions of the heart and skeletal muscle of Calomys callosus infected with the strains $Y$ and 21 SF. A: $Y$ strain - heart section, 20 days after infection, showing moderate interstitial infiltration with mononuclear cells, 200X; B: Y strain - heart section, 40 days of infection, showing focal residual infiltrate, 400X; C: $21 S F$ strain - heart section, 20 days after infection, showing focal necrosis of cardiac myocells and focal mononuclear infiltration, 200X; D: $21 S F$ strain - skeletal muscle, 40 days of infection, focal perivascular, residual mononuclear infiltration, 200X 

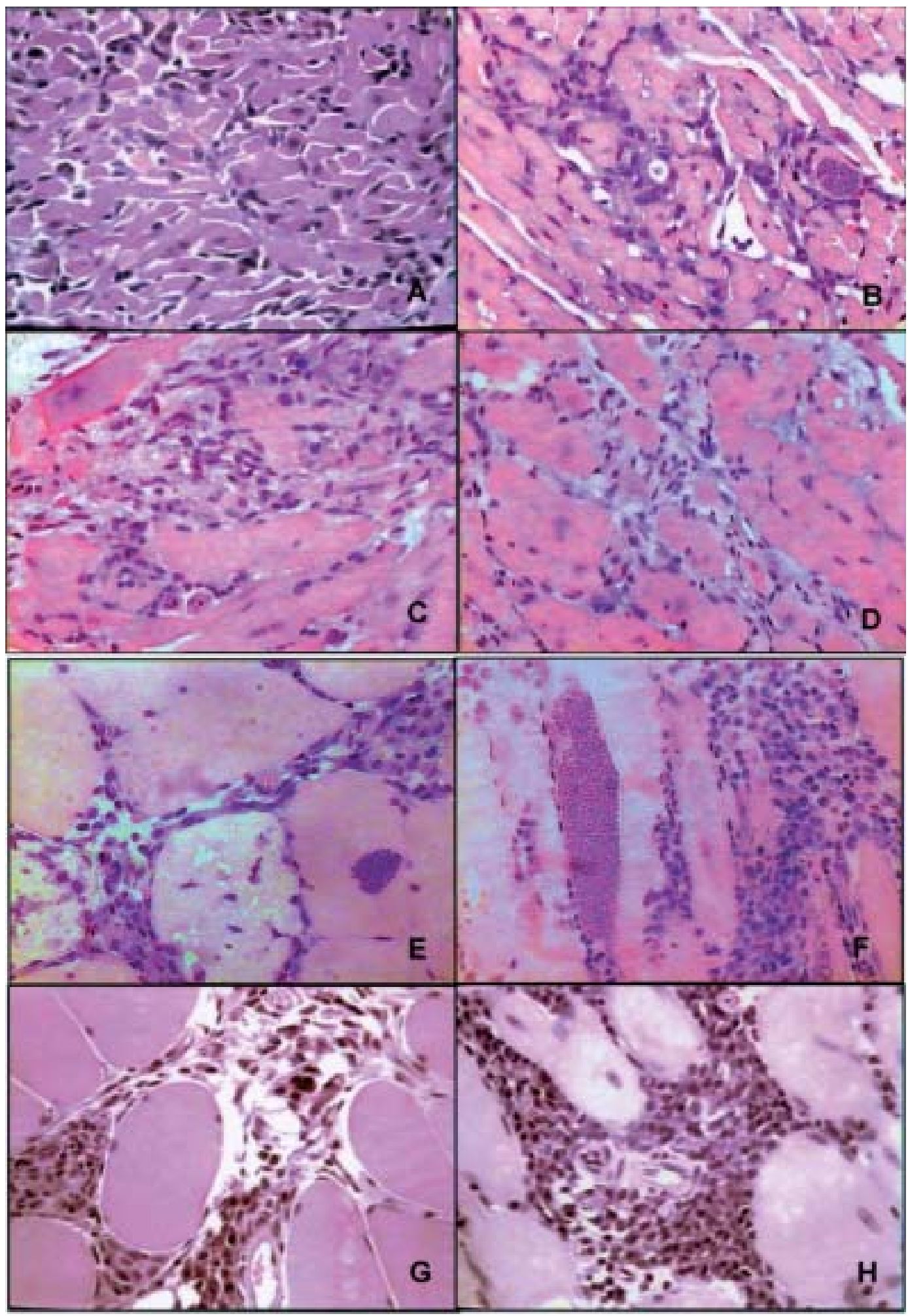

Fig. 3: histopathological lesions of the heart and skeletal muscle of Calomys callosus infected with the Colombian strain - Sections of the myocardium. A: 15 days of infection, interstitial mononuclear infiltration and fibroblasts proliferation, 200X; B: 20 days, focal and diffuse mononuclear infiltration, fibroblasts proliferation and presence of intracellular amastigotes. 400X; C: 25 days, focal disintegration of heart cells with substitution by scarce inflammatory infiltrate and matritial deposits. 400X; D: 35 days, interstitial mononuclear infiltration, fibroblasts proliferation and scarce fibrilar matritial deposits. 400X. Skeletal muscle sections. E: 15 days of infection, intracellular collections of amastigotes of Trypanosoma cruzi without reaction; interstitial focal infiltration with macrophages and proliferation of fibroblasts, 400X; F: 25 days, focal necrosis of muscle cells with intense mononuclear inflammatory infiltration, presence of polymorphonuclear neutrophils, cellular and parasite debris, 400X; G: 30 days, interstitial inflammatory infiltration, with fibroblasts proliferation and collagen deposits, forming strands in the interstitium, 400X; H: 35 days, dense interstitial and perivascular mononuclear infiltration, 400X 
der bundles and peri-vascular thickening. Parasites were scarce in the heart. At 40th day, small foci of destroyed cardiac cells, with focal mononuclear inflammation were present. Picro-Sirius staining for collagen showed fragmentation and a progressive decrease of collagen until the 60th to the 70th day with residual foci of inflammation.

Skeletal muscles - Form 15 to 20 days, lesions of the skeletal muscles were mild and focal, with the presence of intracellular amastigotes, without inflammation, with focal destruction of parasitized muscle cells (Fig. 3E) The lesions became more intense in the skeletal muscle from 25 to 30 days of infection, with focal necrosis of parasitized myocells and the presence of large collections of amastigotes. Intense inflammatory process was present, with predominance of macrophages, lymphocytes and fibroblasts with parasitic debris and polymorphonuclear neutrophils (Fig. 3F,G). In the 35th day of infection, intense interstitial infiltrates were present (Fig. 3H). Strands of collagens were seen in the inflammatory foci (Fig. 3H) and confirmed in the sections stained with Picro Sirius. At the 40th day of infection, the inflammatory lesions became less intense. From 60 to 70 days they were limited to focal areas. Fine strands of collagen appeared in sections stained with Picro Sirius.

Normal controls - Sections of the heart showed normal cardiac structure, with scarce interstitial matrix. Skeletal muscle sections showed characteristic histological structure with a distinct and fine perimisial matrix deposit seen with the Picro Sirius red staining.

\section{Morphometric evaluation of the inflammation}

Myocardium - Results of quantitative morphometric evaluation of the number of inflammatory cells in five areas of inflammation of $12 \mathrm{~mm}^{2}$ in the myocardium are shown in Fig. 4A, revealing significant increasing of the number of inflammatory cells from the 25 th to the 30 th day of infection, followed by a significant decrease from the 40th to 60th day post infection ( $\mathrm{p}<0.01)$.

Skeletal muscle - Fig. 4B shows the quantitative evaluation of the inflammatory infiltrates in the skeletal muscle, with significant increase of the number of cells from 25 to 30 days of infection and decrease from 40 to 60 days ( $p<$ 0.01).

Morphometric evaluation of the fibrosis

Myocardium, Fig. 5A - The morphometric analysis of fibrosis showed slight increase in collagen deposits, as measured in 5 areas of $12 \mathrm{~mm}^{2}$ with decrease to normal levels by the 60th day. Statistical analysis did not show significance when compared with normal controls.

Skeletal muscle, Fig. 5B - Significant increase of collagen deposits was detected by the 30th to 40th days of infection, and show significant decrease in the 60th day as compared to normal controls $(\mathrm{p}<0.01)$.

\section{DISCUSSION}

Findings of the present study indicate clear differences in $C$. callosus response to infection by strains of $T$. cruzi from different biodemes, regarding the degree of the inflammatory process, parasite histotropism, the intensity of parasitism and the evolution of fibrogenesis. Furthermore, the $\mathrm{Y}$ and 21SF strains did not induce a significant

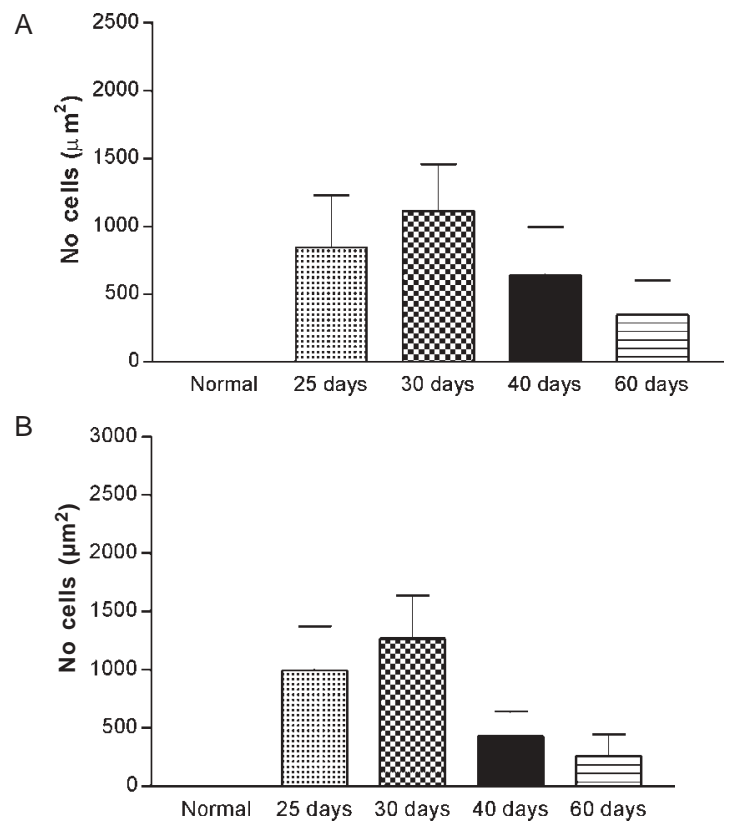

Fig. 4: morphometric evaluation of the inflammatory infiltration in the heart and skeletal muscle of Calomys callosus infected with the Colombian strain of Trypanosoma cruzi. A: heart, showing significant increase of the number of inflammatory cells, compared with normal control, from the 25 th to the 30 th day of infection, followed by a significant decrease from the 40th to 60th day post infection ( $\mathrm{p}<0.01)$; B: skeletal muscle, with significant increase of the number of cells from 25th to 30th days of infection and decrease from 40th to 60th days $(\mathrm{p}<0.01)$

increase of the interstitial matrix components. In contrast, the Colombian strain induced an intense inflammatory process in the heart and skeletal muscles. Significant fibrosis, in the skeletal muscle, that spontaneously subsided during the subacute phase of infection (40 to 60 days) has also been detected. The Colombian strain (Type III) is more pathogenic for the C. callosus than types I (Y) and II (21SF) strains, which determined a mild infection, with an early tendency towards the regression of the lesions, and null animal mortality. Histotropism for the myocardium of the three biodemes was maintained in $C$. callosus, as compared with mice. Presence of amastigotes of $T$. cruzi in cardiac myocells was detected, although with a low degree of parasitism with the $Y$ and 21SF strain. However the macrophagotropism that is a hallmark of the infection with Type I strains in mice, was scarce in the spleen of C. callosus (data not shown). On the contrary, the tropism for skeletal muscles, characteristic of the Colombian strain, was very intense in the $C$. callosus, maintaining the same aspects as seen in mice. The $C$. callosus apparently controls the infection with the Types I and II strains, with inhibition of parasite multiplication and early regression of inflammation, as well as absence of significant fibrogenesis.

The Colombian strain, differently from the others, showed an exacerbation of virulence, expressed by a precocious increasing of the parasitemia differing from the murine model that shows late peaks, from 25 to 30 days. 


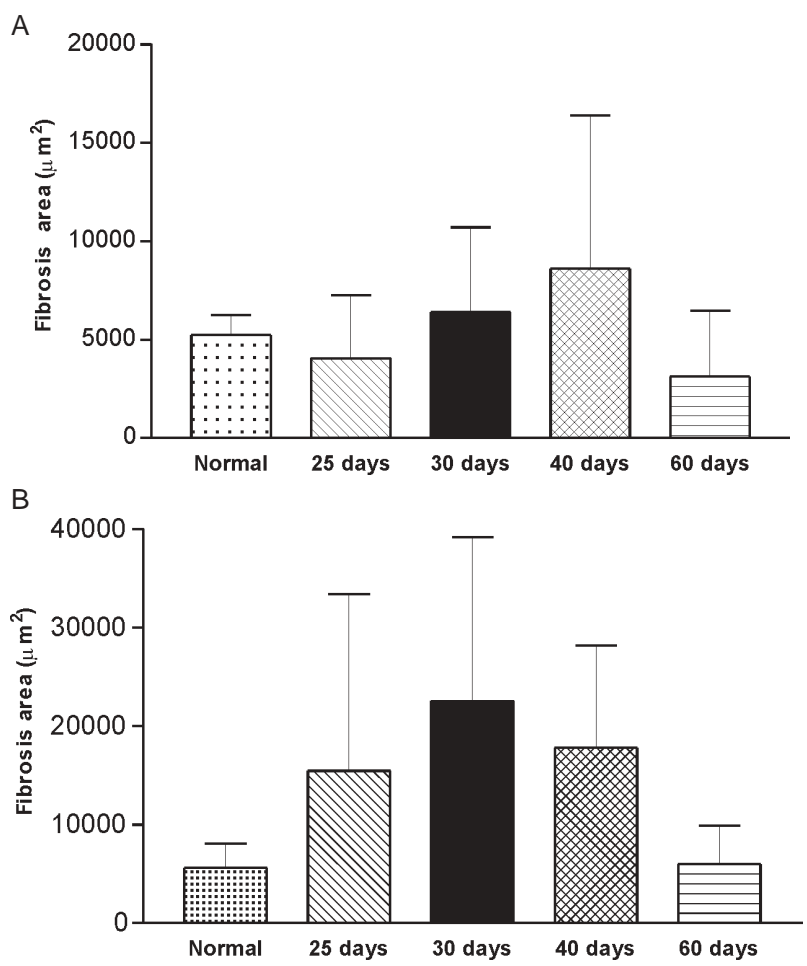

Fig. 5: morphometric evaluation of the collagen deposits in the heart and skeletal muscle of Calomys callosus infected with the Colombian strain of Trypanosoma cruzi: A: heart, although an increase of the fibrosis has been detected in the 40th day, statistical analysis did not show significance when compared with normal controls ( $\mathrm{p}>0.05$ ); B: skeletal muscle, significant increase of collagen deposits by the 30th to 40th days of infection and significant decrease in the 60th day as compared to normal controls $(\mathrm{p}<0.01)$

Also, an increased pathogenicity was detected in $C$. callosus as compared with the murine model. Extensive muscle necrosis and intense inflammatory and fibrotic lesions, were present in C. callosus, confirming the importance of the interaction host/parasite in the patterns of the lesions.

Apparently, several factors, dependent on the parasite strain and on the host response, are responsible for the characteristic lesions in this model. The Colombian strain shows a peculiar behavior in respect to several parameters. The antigenic analysis (Andrade et al. 1981) of the three types of strains showed clear cut differences in their antigenic profiles. Cross reacting antigens were present in the three strains, but Colombian strain has in addition, its own antigenic determinants and this may correspond to differences in its immunogenicity.

The specific neuraminidase activity (Pereira \& Hoff 1986), can influence the predominant tropism for the skeletal myocells (Libby et al. 1986). Pereira and Hoff (1986), studying the neuraminidase activity of several strains, have shown higher activity of neuraminidase in the Colombian strain as compared with Y and 12 SF strains, which could be associated with the myotropism of the Colombian strain. The infective trypomastigote forms of T. cruzi exhibit neuroaminidase activity and can desialylate cardiac cells in culture. These observations are in accordance with those of Shenkmann et al. (1991, 1992), who identified the transialidase that transfer the sialic acid from the surface of host cells to the surface of the parasite, and participates in the penetration of trypomastigotes into the host cells. These aspects are important but do not exclude the participation of the host response. The genetic types of collagen investigated in a previous study (Magalhães-Santos et al. 2002) do not influence the regression of the collagen deposits. Differing from $C$. callosus, evolution of the fibrogenesis in the murine model is slow and progressive until the chronic phase of infection (150 to 200 days post-infection). Reversion of fibrosis in mice occurred after specific treatment in the chronic phase of infection, but it did not occur spontaneously (Andrade et al.1991).

C. callosus apparently controls the infection with strains of T. cruzi of BiodemesTypes I and II, being more susceptible to the strains of sylvatic origin represented by the Biodeme Type III (T. cruzi I).

The results confirm the importance of the different biodemes in the determination of tissue lesions and the peculiarities of response of $C$. callosus to infection with T. cruzi.

\section{ACKNOWLEDGMENTS}

To the biologist Barbara Cristina Alves Assis, for her assistance in the morphometric analysis.

\section{REFERENCES}

Andrade SG 1974. Caracterização de cepas do Trypanosoma cruzi isoladas no Recôncavo Bahiano. Rev Pat Trop 3: 65121.

Andrade SG, Magalhães JB 1997. Biodemas e zimodemas of $T$. cruzi strains: correlations with clinical data and experimental pathology. Rev Soc Bras Med Trop 30: 27-35.

Andrade SG, Andrade V, Rocha Filho FD, Barral Netto M 1981. Análise antigênica de diferentes cepas do T. cruzi. Rev Inst Med Trop São Paulo 23: 245-250

Andrade SG, Kloetzel JK, Borges MM, Ferrans VJ 1994. Morphological aspects of the myocarditis and myositis in Calomys callosus experimentally infected with Trypanosoma cruzi : fibrogenesis and spontaneous regression of fibrosis. Mem Inst Oswaldo Cruz 89: 379-393.

Andrade SG, Stocker-Guerret S, Pimentel AS, Grimaud JA 1991. Reversibility of cardiac fibrosis in mice chronically infected with Trypanosoma cruzi, under specific chemotherapy. Mem Inst Oswaldo Cruz, 86: 187-200.

Andrade V, Andrade SG, Barral Netto M, Pontes AL, Castro R 1985. Avaliação do comportamento de diferentes cepas do T. cruzi na infecção de seis linhagens isogênicas de camundongos. Rev Soc Bras Med Trop 18: 143-154.

Borges MM, Andrade SG, Pilatti CG, Prado-Jr JC, Kloetzel JK 1992. Macrophage activation and histopathological findings in Calomys callosus and Swiss mice infected with several strains of Trypanosoma cruzi. Mem Inst Oswaldo Cruz 87: 493-502.

Brumpt E, Da Silva P 1912. Existence du "Schyzotrypanum cruzi” Chagas, 1909, à Bahia (Matta de São João). Biologie du "Conorrhinus megistus". Bull Soc Path Exotique 5: 2226.

Chagas C 1909. Nova tripanosomiase humana. Mem Inst Oswaldo Cruz 1: 159-218. 
Junqueira LCU, Bignolas G, Bretani RR 1979. Picrosirius staining plus polarization microscopy, a specific method for collagen detection in tissue sections. Histochem J 11: 447455.

Libby P, Alroy J, Pereira MEA 1986. A neuraminidase from Trypanosoma cruzi removes sialic acid from the surface of mammalian myocardial and endothelial cells. J Clin Invest 77: 127-135.

Magalhães JB, Pontes AL, Andrade SG 1985. Comportamento das cepas Y e Peruana do Trypanosoma cruzi no camundongo, após passagens em diferentes meios. Mem Inst Oswaldo Cruz 80: 41-50.

Magalhães-Santos IF, Lima ES, Andrade SG 2002. Fibrogenesis and collagen resorption in the heart and skeletal muscle of Calomys callosus experimentally infected with Trypanosoma cruzi: immunohistochemical identification of extra- cellular matrix components. Mem Inst Oswaldo Cruz 97: 703-710.

Momem H 1999. Taxonomy of Trypanosoma cruzi: a commentary on characterization and nomenclature. Mem Inst Oswaldo Cruz 94: 181-184.

Pereira MEA, Hoff R 1986. Heterogeneous distribution of neuraminidase activity in strains and clones of Trypanosoma cruzi and its possible association with parasite myotropism. Mol Biochem Parasitol 20: 183-189.

Schenkman S, Carvalho LP, Nussenzweig V 1992. Trypanosoma cruzi trans-sialidase and neuraminidase activities can be mediated by the same enzymes. J Exp Med 175: 567575.

Schenkman S, Jiang MS, Hart GW, Nussenzweig V 1991.A novel cell surface trans-sialidase of Trypanosoma cruzi generates a stage specific epitope required for invasion of mammalian cells. Cell 65: 1117-1125. 
